\title{
Is It Muscle Confusion or Cross Training?
}

\section{Geffrey Colón, PhD}

Professor, College of Health and Human Services, Eastern Michigan University Ypsilanti, MI 48197, United States.

Article Details
Article Type: Research Article
Received date: $05^{\text {th }}$ November, 2021
Accepted date: $11^{\text {th }}$ December, 2021
Published date: $13^{\text {th }}$ December, 2021
Corresponding Author: Geffrey Colón, PhD, Professor, College of Health and Human Services, Eastern Michigan University
Ypsilanti, MI 48197 , United States.E-mail: gcolon@emich.edu
Citation: Colón, G. (2021). Is It Muscle Confusion or Cross Training?. J Pub Health Issue Pract 5(2): 191. doi: https://doi.
org/10.33790/jphip1100191
Copyright: C2021, This is an open-access article distributed under the terms of the Creative Commons Attribution License
4.0, which permits unrestricted use, distribution, and reproduction in any medium, provided the original author and source
are credited.

\begin{abstract}
This article is a commentary on recommendations to maintain a seasonal physically active lifestyle for individuals that reside in geographical areas exposed to changing weather patterns. The concepts of muscle confusion and cross-training are explained including the benefits for each of the training regimens. Benefits include avoidance of overuse injuries, overtraining, burnout, and increased motivation to maintain a lifestyle with physical activity. Suggestions applicable to the State of Michigan are mentioned but such are applicable to other areas of the world with similar weather seasons that have periodic changes.
\end{abstract}

Key words: Seasonal Physical Activity, Resistance Training, Injury Avoidance, Barre Fitness, Motivation

\section{Introduction}

The TV/Radio goes-on and infomercials are simply telling us that we can get in the best shape of our lives if we purchase programs such as P90X, 10-Minute-Workout, Insanity, and/or many others. These programs do produce significant results if followed according to the prescription of the creators and a sensible nutritional program accompanies the fitness program. Credit must be given to the timing and smart advertising as well as to the creators of such programs because they have capitalized on a principle that is not new to exercise science [1]. Part of the hype for such programs is the principle of muscle confusion. Muscle confusion is based on varying the types of stress that are placed on the muscular system to avoid muscle accommodation over time because of repetition of the same exercises [2]. If exercises are not varied, then the body becomes usedto-the-same exercise and a level of complacency ensues because the muscles are "comfortable" with the repetitive exercise [3]. Muscle confusion is often connected to strength training and body building for the purpose of hypertrophy. However, in this article I apply the term to exercise variations throughout the year that bring positive benefits to the musculoskeletal system and the general well-being of the individual.

Needless to say, all of us have our favorite exercises and sports and we look forward to being physically active in order to maintain our level of fitness. Consequently, especially because of motivation and "love" for the physical activity that we have done over the years stopping such exercise or sport is "normally" not an option for many of us (unless for example, overtraining, burnout, age, or injury dictate we stop). So, if we are faithful to a specific fitness routine, how do we adjust to include the aspect of muscle confusion in a manner that does not detract from motivation and/or doing a sport we enjoy?
My answer to such dilemma is cross-training, and for us in Michigan, seasonal cross-training is an added benefit. Cross-training goes as far back as the Ancient Greeks, according to Godfrey [4], Philostratos in the period of $\mathrm{AD} 170-249$ recommended athletes to engage in different fitness activities such as endurance runs, weight training, and wrestling animals to achieve high levels of physical fitness.

Engaging in a cross-training fitness regimen means we are varying the fitness exercises and/or sport on a regular schedule in order to keep our muscles active with different types of movements to stay in-shape [5]. In other words, because we are engaging in different activities, our muscles are performing different motions and the concept of muscle confusion is being achieved even if we are unaware that such principle is fully engaged by the switch in activities. For us in Michigan the seasons lend themselves to perfect cross -training scenarios that can only serve to maintain motivation for being active, and for doing activities conducive to each season.

In my case, one constant across the year is resistance training for muscle endurance but I periodically vary how I work the principle of muscle overload. I tend to focus in 5 different aspects of resistance training, using machines, free-weights with a bar, dumbbells, elasticfitness-bands, and traditional body-weight exercises or with newer methods such as TRX Bands. I also discovered the benefits of barre fitness classes and I have become a regular participant in this fitness activity. Barre fitness provides a number of added benefits such as core strength, static/dynamic balance, muscle endurance, coordination, and flexibility. Not to mention using muscle groups not normally used in many sporting activities [6]. All of these exercise variations bring aspects of muscles being used slightly differently and the body is not getting complacent with the same range of motion.

What I do vary seasonally are my sporting activities. In late fall and winter my focus is on playing basketball, volleyball, racquetball, and snowboarding. In spring and summer golf takes over, however, I am more prone to walk and carry my bag as that is how golf was meant to be played in my mind and it becomes a more comprehensive exercise session. Summer is my favorite time of the year, so hiking, walking/running outdoor trails, swimming, water-sports (knee/ wake-boarding, boogie boarding), kayaking, and bicycling tend to dominate the physical activity schedule. My list of activities is varied and some activities require more effort/skill than others, but at the end I am still being active and engaging muscles differently. Thus, by means of the cross-training approach muscles are on constant alert accommodating to the correct range of motion in order to perform the desired movement. 
Besides engaging muscles differently with a cross-training approach other benefits come along with it. By switching activities periodically, the body is less prone to the overuse nagging injuries (you know, the ones that hurt enough...but not enough to stop the activity) caused by repetition and the wear/tear on the muscle/joint because of the same movement [7]. Another benefit is a higher motivation to stay physically active because cross-training offers a variety of beautiful and physically engaging settings, social connections, and a challenge to perform according to your own personal standards per activity [8].

In order to benefit from a cross-training fitness regimen, you have to be willing to try new activities and find the "passion" for new forms of exercise. Venture out to take lessons or ask a friend to teach you "their sport or physical activity" which intrigues you, and give yourself the chance to determine which new activities you like and do not like. Your approach to seasonal cross-training will give you the benefits of muscle confusion while being active in exercises that provide the sense of personal satisfaction for staying physically fit throughout the entire year.

Conflict of interests: The author declares no conflict of interest.

\section{References}

1. Venuto, T. (2012). The Truth About Muscle Confusion Training. Iron Magazine. June, 2012. The Truth About Muscle Confusion Training | IronMag Bodybuilding Blog (ironmagazine.com)

2. Mawer, R. (2018). Is Muscle Confusion the Right Move. Is Muscle Confusion The Right Move? (rudymawer.com)
3. Lipowski, M. (2014). Exercise is a Must, Muscle Confusion is Moronic. The Art of Muscle Strength and Science. Mt. Kisco | Exercise | Fitness | Bodybuilding (mikelipowski.com)

4. Godfrey, R.J. (1998). Cross-Training. Journal of Sport and Injury. Volume 4, Pages 50-55. https://www.researchgate.net/ profile/Rj-Godfrey/publication/292511523_Cross-training/ links/575000ab08ae5c7e547a8602/Cross-training.pdf

5. O'Keefe, E.L. \& Lavie, C.J. (2020). A Hunter-Gatherer Exercise Prescription to Exercise and Well-Being in the Modern World. Journal of Science in Sport and Exercise volume 3, pages147-157 (2021). A Hunter-Gatherer Exercise Prescription to Optimize Health and Well-Being in the Modern World | SpringerLink

6. Burcham, C. \& Rostamian, M. (2021). 8 Things That Happen to Your Body When You Start Doing Barre. Byrdie August 25th, 2021. https://www.byrdie.com/barre-benefits

7. Gaz, D.V. \& Smith, A.N. (2012). Psychosocial Benefits and Implications of Exercise. $P M \& R$ Volume 4, Issue 11, November 2012, Pages 812-817. Psychosocial Benefits and Implications of Exercise - ScienceDirect

8. Nied, R.J. \& Franklin, B. (2002). Promoting and Prescribing Exercise for The Elderly. Am Fam Physician. 2002 Feb 1;65(3):419-427. Promoting and Prescribing Exercise in the Elderly - American Family Physician (aafp.org) 